\title{
Laser lithotripsy resolution for Bouveret syndrome
}

A 75-year-old woman was admitted with epigastric pain, nausea, and vomiting, but without any other symptoms. Laboratory testing and physical examination were normal. Her abdominal computed tomography (CT) scan on admission identified a cholecystoenteric fistula (duodenum to distal antrum) with a 4-cm stone in the duodenum, all of which was compatible with Bouveret syndrome ( Fig.1) We performed a gastroscopy, which identified pyloric obstruction due to a huge stone that was preventing advancement of the endoscope ( $\triangleright$ Fig. 2 a). The patient was discussed with the surgical department, following which conservative management involving endoscopic removal was chosen.

An endoscope was inserted and we identified the presence of a large stone that was nestled in the bulb and just visible in the antrum through the pylorus. After various different manipulations using a Roth net had been tried unsuccessfully, the stone was fragmented with a lithotripsy laser (Holmium probe, 27 microns) without incident ( $>$ Fig.2 b). All of the small fragments were removed with forceps and a Roth net ( $\boldsymbol{F i g} \cdot \mathbf{2 c}$ ). Finally, we explored the bulb and noted ulceration on the posterior side, as well as the cholecystoduodenal fistula ( $\triangleright$ Fig. 2 d; - Video 1). The area was washed thoroughly, and once we had confirmed that the stone had been completely removed, the procedure was finished. The patient was discharged after 3 days with no further symptoms.

Bouveret syndrome is a rare cause of intestinal obstruction that predominantly affects elderly women with multiple pathologies [1-3]. New endoscopic techniques are promising because they offer less risk than surgery and with great efficiency [3-5]. Some mechanical methods, such as mechanical lithotripsy or the novel use of endoscopic laser lithotripsy, are now being used to destroy large stones with less morbidity and good outcomes [3-5].

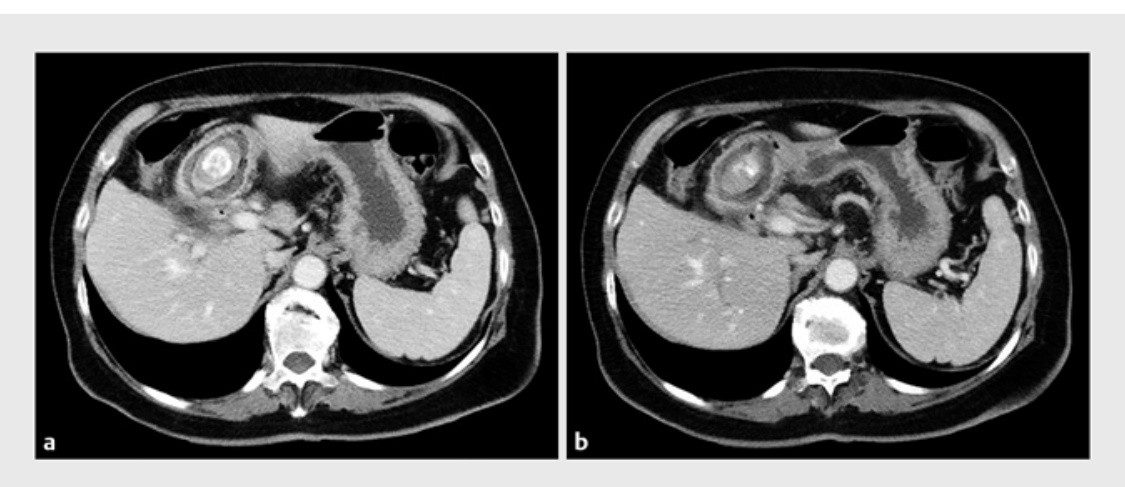

- Fig. 1 Computed tomography (CT) scan images showing: a the stone located in the duodenum; $\mathbf{b}$ the cholecystoenteric fistula.

Endoscopy_UCTN_Code_TTT_1AR_2AH The Authors

Competing interests

None
Cristina Saldaña Dueñas, Ignacio FernándezUrien, María Rullán Iriarte, Juan José Vila Costa

Gastroenterology Department of "Complejo Hospitalario de Navarra”, Pamplona, Spain

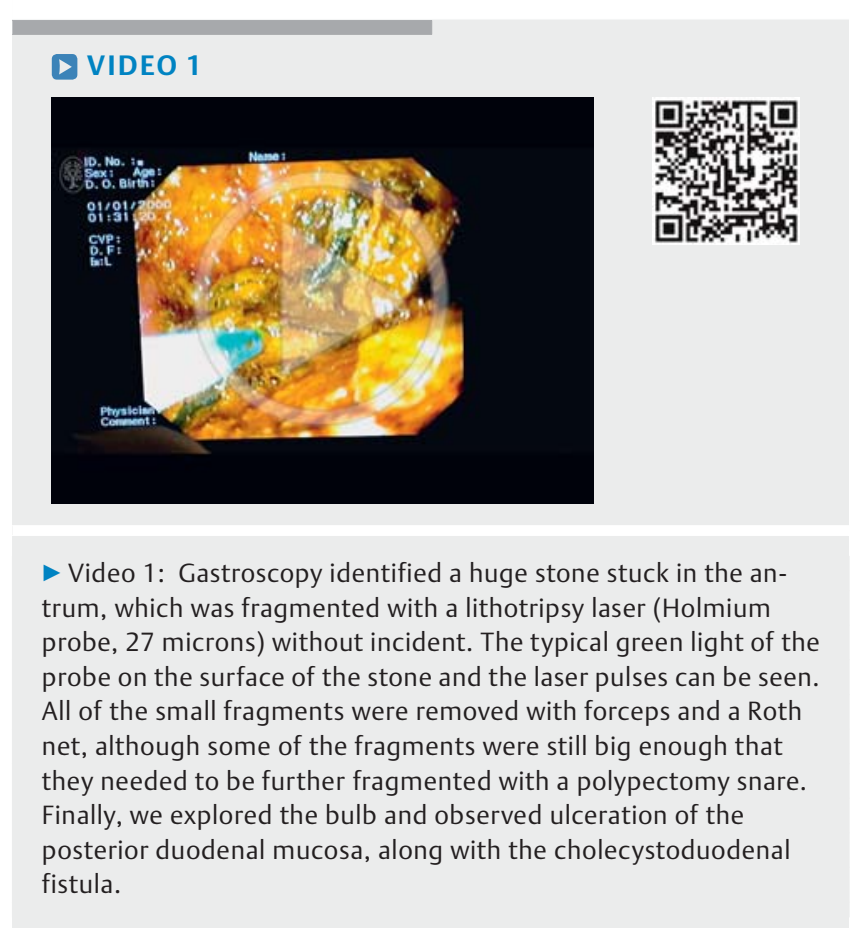




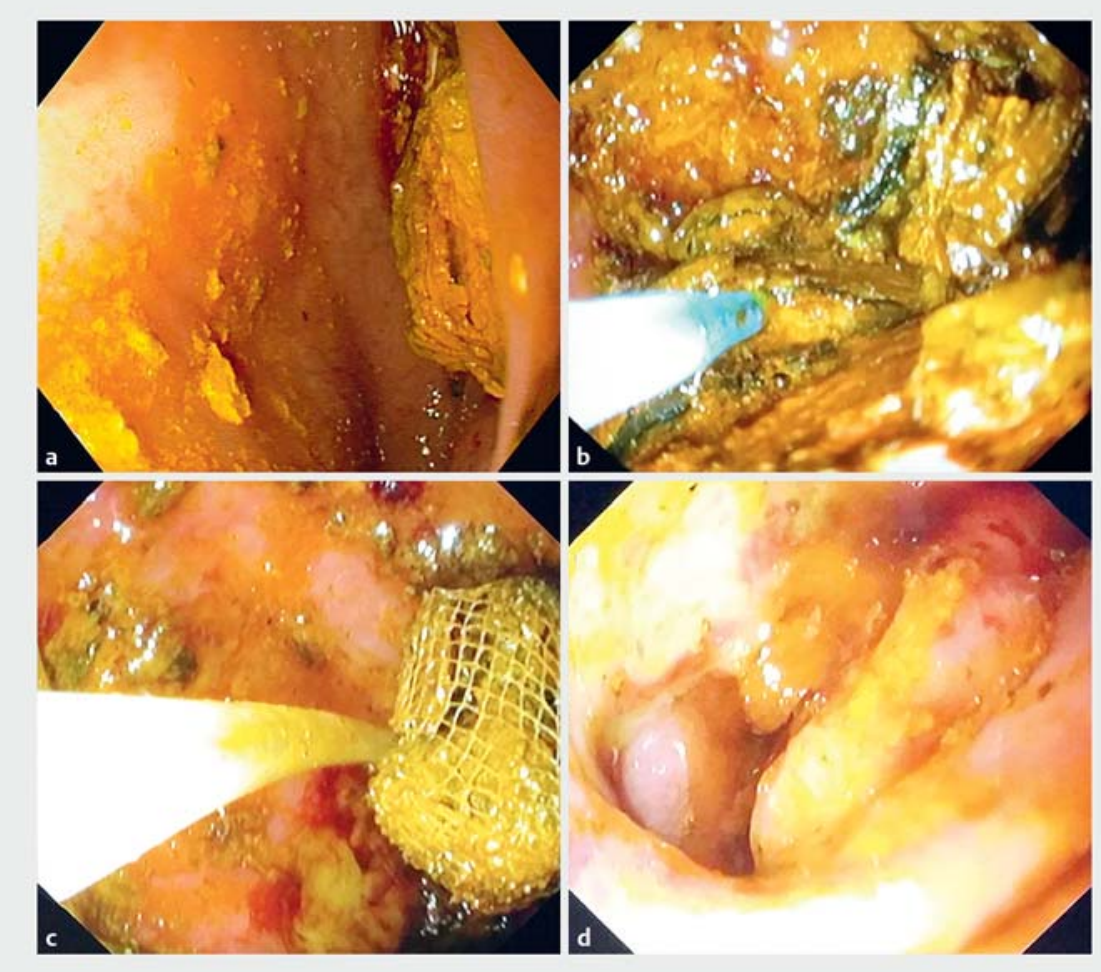

- Fig. 2 Endoscopy images showing: a the stone stuck in the pyloric area; $\mathbf{b}$ laser lithotripsy fragmentation (note: the typical green light on the surface of the stone); $c$ stone fragments being removed in a Roth net; $\mathbf{d}$ ulceration of the duodenal mucosa and the cholecystoduodenal fistula.
Corresponding author

\section{Cristina Saldaña Dueñas, MD}

Gastroenterology Department of "Complejo Hospitalario de Navarra", Calle Irunlarrea n³ sn 31008, Pamplona, Spain

crisaldu@hotmail.com

\section{References}

[1] Cappell MS, Davis M. Characterization of Bouveret's syndrome: a comprehensive review of 128 cases. Am J Gastroenterol 2006; 101: $2139-2146$

[2] Lawther RE, Diamond T. Bouveret's syndrome: gallstone ileus causing gastric outlet obstruction. Ulster Med J 2000; 69: 69-70

[3] Makker J, Muthusamy VR, Watson R et al. Electrohydraulic lithotripsy and removal of a gallstone obstructing the duodenum: Bouveret syndrome. Gastrointest Endosc 2015; 81: 1021-1022

[4] Sethi S, Kochar R, Kothari S et al. Good vibrations: successful endoscopic electrohydraulic lithotripsy for Bouveret's syndrome. Dig Dis Sci 2015; 60: 2264-2266

[5] Rivera Irigoín R, Ubiña Aznar E, García Fernández $G$ et al. [Successful treatment of Bouveret's syndrome with endoscopic mechanical lithotripsy]. Rev Esp Enferm Dig 2006; 98: 790 - 792

\section{Bibliography}

DOI http://dx.doi.org/10.1055/s-0043-102178

Endoscopy 2017; 49: E101-E102

(c) Georg Thieme Verlag KG

Stuttgart · New York

ISSN 0013-726X 